\title{
Estudio sobre el consumo de riesgo y perjudicial del alcohol en alumnos de la UT del Norte de Aguascalientes
}

\section{Study on the risk and harmful consumption of alcohol in students of the UT of North Aguascalientes}

\author{
DEL VALLE-ÁVILA, Paula Carmina*† \& GARCÍA-RODRÍGUEZ, Juan Carlos \\ Universidad Tecnológica del Norte de Aguascalientes, Av. Universidad No.1001, Estación Rincón, Rincón de Romos, Ags. \\ C.P. 20400
}
ID $1^{\mathrm{er}}$ Autor: Paula Carmina, Del Valle-Ávila / ORC ID: 0000-0002-7852-7713, Researcher ID Thomson: V-4804-2018, CVU CONACYT ID: 953424

ID $1^{\text {er }}$ Coautor: Juan Carlos, García-Rodríguez / ORC ID: 0000-0002-3602-7809, Researcher ID Thomson: J-8291-2017, CVU CONACYT ID: 677265

\section{Resumen}

En este artículo se expone un estudio sobre el consumo de riesgo y perjudicial del alcohol en jóvenes de la Universidad Tecnológica del Norte de Aguascalientes, el cual tiene por objetivo el hacer un diagnóstico situacional sobre los hábitos de consumo de alcohol, así como su nivel de riesgo, consumo perjudicial y posible dependencia en dicha población, para tal fin se utilizó como instrumento el Test de Identificación de los Trastornos Debidos al Consumo de Alcohol (Alcohol Use Disorders Identification Test, AUDIT), el cual fue aplicado de manera digital en 756 alumnos de segundo cuatrimestre en 14 programas educativos del nivel Técnico Superior Universitario, de esta forma se identificó el tipo de consumo de alcohol, lo que permitirá a la Universidad implementar acciones para la sensibilización y concientización sobre las consecuencias negativas de su consumo en los diferentes ámbitos de su vida como lo son el académico, manifestado a través del bajo desempeño escolar, rezago y/o deserción, así como en el área personal, social y familiar.

\section{Alcohol, Consumo, Diagnóstico}

\begin{abstract}
This article presents a study about the risk and harmful consumption of alcohol in young people of the Technological University of North Aguascalientes, whose objective is to make a situational diagnosis about the habits of alcohol consumption, as well as their level of risk, harmful consumption and possible dependence in said population, for this purpose the Identification Test of Alcohol Consumption Disorders (Alcohol Use Disorders Identification Test, AUDIT) was used as an instrument, which was applied digitally in 756 students of second semester in 14 educational programs of the Higher University Technical level, in this way the type of alcohol consumption was identified, which will allow the University to implement actions to raise awareness and awareness of the negative consequences of its consumption in the different areas of his life as the academic is, manifested through the low scholastic performance, lag and / or desertion, as well as in the personal, social and family area.
\end{abstract}

Alcohol, Consumption, Diagnosis

Citación: DEL VALLE-ÁVILA, Paula Carmina \& GARCÍA-RODRÍGUEZ, Juan Carlos. Estudio sobre el consumo de riesgo y perjudicial del alcohol en alumnos de la UT del Norte de Aguascalientes. Revista de Gestión Universitaria. 2019. 3-9: 2131

*Correspondencia del Autor (correo electrónico: paula.avila@utna.edu.mx)

$\dagger$ Investigador contribuyendo como primer autor. 


\section{Introducción}

En México el consumo de alcohol ha ido en aumento en la población más joven, de acuerdo a datos emitidos por la Comisión Nacional Contra las Adicciones (2019) citado por Rodríguez (2019), la edad de inicio se da a partir de los diez años, lo cual representa un grave problema, ya que hay una alta probabilidad de que en edades muy tempranas puedan presentar una dependencia al alcohol (Rodríguez, 2019).

En Aguascalientes, de acuerdo a datos del Instituto Mexicano del Seguro Social, el consumo de alcohol comienza en más del 80 por ciento de los jóvenes entre 14 y 20 años de edad, y según la Encuesta Nacional de Consumo de Drogas en Estudiantes 2014, el Estado está por encima de la media nacional en el porcentaje de los jóvenes estudiantes de secundaria y bachillerato que requieren tratamiento debido a adicciones (La Jornada de Aguascalientes, 2017).

Es en las Instituciones de Educación desde nivel básico hasta superior donde se pueden realizar acciones de prevención; sensibilizando y concientizando a los niños y adolescentes sobre los riesgos del consumo y abuso del alcohol, así como implementar estrategias para una oportuna detección y canalización a las instancias correspondientes para su tratamiento.

La Universidad Tecnológica del Norte de Aguascalientes, a través del área de Asesoría Psicopedagógica, ha detectado algunas problemáticas en la población estudiantil que están relacionadas con el consumo y abuso del alcohol, como lo es el ausentismo, bajo desempeño académico, embarazos no planeados, accidentes automovilísticos, riñas e incluso la muerte. Por tal razón, es importante tener un estudio sobre las conductas de consumo en dicha población, lo cual permita implementar estrategias de prevención e intervención.

Con el presente estudio se pretenden conocer sí hay un consumo perjudicial y posible dependencia al alcohol en jóvenes universitarios de esa casa de estudios.

\section{Objetivo}

El objetivo de este estudio es identificar el consumo de riesgo y perjudicial del alcohol en alumnos de la UT del Norte de Aguascalientes.

\section{Antecedentes}

El problema del alto consumo del alcohol está presente en los diversos estratos de la sociedad, incluyendo a los estudiantes de nivel universitario. A estos últimos consumidores, los afecta tanto en los aspectos físicos ocasionados por el consumo, como en aspectos académicos de los mismos.

A nivel nacional, la Encuesta Nacional de Consumo de Drogas, Alcohol y Tabaco (ENCODAT) 2016-2017, arroja resultados que merecen ser comentados, ya que muestran las tendencias en cuanto a consumo de alcohol en nuestro país.

Dentro de los datos más relevantes, dicha encuesta muestra en resumen que el consumo de alcohol "alguna vez en la vida" se ha mantenido estable con relación al 2011 (71.3\% a 71\%). La prevalencia del "último año" disminuyó de $51.4 \%$ a $49.1 \%$ y para "último mes" incrementó de $31.6 \%$ a $35.9 \%$. En los hombres, la prevalencia de consumo "último mes" aumentó de $44.3 \%$ a $48.1 \%$, y en las mujeres pasó de $19.7 \%$ a $24.4 \%$.

El "consumo excesivo en el último mes" en la población total incrementó de $12.3 \%$ a $19.8 \%$ en comparación a 2011, mientras que para la población de 18 a 65 años pasó de $13.9 \%$ a $22.1 \%$.

Tanto el "consumo diario" como "consuetudinario" han incrementado de $0.8 \%$ a $2.9 \%$ y de $5.4 \%$ a $8.5 \%$ respectivamente en la población total; en la población de 18 a 65 años pasó de $0.9 \%$ a $3 \%$ y de $6.3 \%$ a $9.3 \%$ en los mismos rubros.

El $0.6 \%$ de las mujeres y el $3.9 \%$ de los hombres reportaron dependencia al consumo de alcohol, mientras que para la población total, el $2.2 \%$ reportó dependencia.

Las personas que han bebido en el último año, indican que necesitaron en promedio 8.7 copas para sentirse borrachos. Por sexo, los hombres indican necesitar 10 copas en comparación con seis que indican las mujeres.

La edad de inicio en el consumo de alcohol fue en promedio de 17.8 años; las mujeres iniciaron a los 19.2 años y los hombres a los 16.7. 
En cuanto al consumo per cápita, la población reportó beber 4.9 litros de alcohol puro, siendo que los hombres consumen más del triple que las mujeres (7.9 litros hombres y 2.1 litros mujeres). La edad de mayor consumo es entre los 18 y 29 años (7.6litros). Los estados de Aguascalientes (8 litros), Jalisco (7.9) y Nuevo León (7.4 litros) reportan mayor consumo per cápita en comparación con el consumo nacional.

La dependencia en el consumo de bebidas alcohólicas dificultó en los hombres su capacidad para trabajar o estudiar en una media de 4.1 en el rango de edad de 18 a 65 años; mientras que en las mujeres, dificultó su vida social y el realizar sus tareas domésticas con medias de 4.0 y 3.5 respectivamente para edades de 12 a 17 años y de 18 a 65 años. El 9.1\% de los hombres y el 3\% de las mujeres ha presentado problemas alguna vez en su vida, en la escuela o trabajo debido a su consumo; en cuanto a los accidentes provocados en el último año por el consumo de alcohol, estos ocurren en mayor medida en hombres $(2.3 \%)$ que en mujeres $(0.5 \%)$.

Los indicadores mostrados ponen de manifiesto la importancia de atender la cuestión del alcoholismo, ya que aunque su consumo se considera legal, este se ha convertido en un problema de salud pública, además de un problema educativo en los jóvenes estudiantes de nivel medio superior y superior, debido a la afectación del consumo de alcohol en la vida estudiantil de cada consumidor, lo cual puede generar problemas educativos como: rezago, bajo desempeño académico, deserción, violencia, bullying, etc.

El Estado de Aguascalientes cuenta con un factor cultural promotor del consumo de alcohol en los jóvenes, el cual es la Feria Nacional de San Marcos, llamada por algunos medios de comunicación como "la cantina más grande del mundo"; esto sin dejar de lado a las múltiples y variadas celebraciones o ferias regionales, en donde los jóvenes ven la oportunidad y sienten la presión por consumir alcohol. El consumo de alcohol debe ser atendido desde la perspectiva institucional, pues esto le permitirá contribuir a que los jóvenes estudiantes no vean disminuidas sus oportunidades de acceso, permanencia y egreso de la UTNA, apoyando a los jóvenes a concluir sus estudios y acceder a mejores niveles de vida, así como incrementar los índices de calidad educativa de la misma universidad.

\section{Marco Teórico}

\section{Alcoholismo}

Existen diversas concepciones acerca del alcoholismo; Bolet (2000) considera que el alcoholismo es una enfermedad crónica, progresiva y fatal cuyas características son la tolerancia y dependencia física, así como cambios orgánicos patológicos, todo debido directa $\mathrm{o}$ indirectamente al alcohol ingerido.

Otaño \& Valdes (2004) mencionan que según el grupo de Clement E., el alcoholismo es un trastorno que padece una persona al ingerir licor con frecuencia y exceso, lo que puede generarle problemas de índole familiar, laboral, social, psicológica, personal y física.

Para el Instituto Mexicano del Seguro Social (IMSS) el alcoholismo es una enfermedad $\mathrm{y}$ no un vicio, cuya dependencia al alcohol deteriora la salud física y mental; quien lo padece manifiesta necesidad de beber alcohol en todo momento y sin control, lo cual lo lleva a realizar cualquier actividad si esta le permite conseguir alcohol, además de mostrarse desinteresado por su salud y aspecto físico (IMSS, 2015).

Según el Dr. David L. Ohlms, citado por Alcohólicos Anónimos (2012), "el alcoholismo es una enfermedad crónica, progresiva e incurable, caracterizada por la pérdida del control sobre el alcohol".

Es así como se puede determinar que el alcoholismo es una dependencia y tolerancia al consumo de alcohol, lo que obliga al alcohólico a la ingesta habitual y constante, en detrimento de su estado físico y psicológico, lo cual puede llevar a consecuencias fatales.

\section{Adicción}

La adicción es definida, por el Dr. Saúl Alvarado (2016) como una enfermedad primaria, crónica con factores genéticos, psicosociales y ambientales. Es frecuentemente progresiva y fatal. Se caracteriza por episodios continuos o periódicos de descontrol en el uso y distorsiones del pensamiento, principalmente la negación. 
Otro doctor, el Dr. Urgelés (2015) establece que las adicciones son enfermedades que afectan a la motivación, entendiendo por motivación las causas (objetivos) de nuestra conducta y nuestras decisiones.

Según la CONADIC (2016), la adicción es la dependencia fisiológica y/o psicológica a alguna sustancia psicoactiva legal o ilegal, provocada por el abuso en el consumo, que causa una búsqueda ansiosa de la misma.

La Organización Mundial de la salud la definió por primera vez en 1964 como un estado de intoxicación periódica o crónica producida por el consumo repetido de una droga natural o sintética.

El IMSS (2016) define adicción al consumo frecuente de estupefacientes, a pesar de saber las consecuencias negativas que producen. Entre otras cosas, modifican el funcionamiento del cerebro y su estructura, provocando conductas peligrosas. Es difícil dejar de consumirlas por las alteraciones en los mecanismos reguladores de la toma de decisiones y del control inhibitorio y porque quien las usa dedica bastante tiempo en su búsqueda y consumo.

\section{AUDIT}

Test de Identificación de los Trastornos Debidos al Consumo de Alcohol (Alcohol Use Disorders Identification Test, AUDIT), fue desarrollado y evaluado a lo largo de un período de dos décadas, habiéndose demostrado que proporciona una medida correcta del riesgo según el género, la edad y las diferentes culturas; este test consta de 10 preguntas, las cuales se dividen en tres dominios, permitiendo encontrar información relevante en cada ítem; los dominios, ítem y preguntas se clasifican de la siguiente forma:

Dominio: Consumo de riesgo de alcohol. Pregunta 1: frecuencia de consumo.

Pregunta 2: cantidad típica.

Pregunta 3: frecuencia del consumo elevado.

Dominio: Síntomas de dependencia.

Pregunta 4: pérdida del control sobre el consumo.

Pregunta 5: aumento de la relevancia del consumo.

Pregunta 6: consumo matutino.

\begin{abstract}
Dominio: Consumo perjudicial de alcohol.

Pregunta 7: sentimiento de culpa tras el consumo.

Pregunta 8: lagunas de memoria.

Pregunta 9: lesiones relacionadas con el alcohol.

Pregunta 10: otros se preocupan por el consumo.
\end{abstract}

Pregunta 10: otrosse preocupan por el consumo.

El AUDIT tiene varias ventajas, como es la estandarización transnacional, pues ha sido validado en seis países, identifica el consumo de riesgo y perjudicial de alcohol, así como una posible dependencia; es breve, rápido y flexible; se centra en el consumo reciente de alcohol (Babor, Higgins-Biddle, Saunders \& Monteiro, 2001). La NOM-028-SSA2-2009 ofrece una versión en español del AUDIT elaborada por De la Fuente J.R., Kershenovich D. El cual se tomó como base para hacer el diagnóstico de esta intervención. A su vez, después del cuestionario, muestra los rangos de evaluación en base a la calificación total obtenida en el cuestionario, los cuales se muestran a continuación:

0 a 7 Abstemio o consumo de bajo riesgo 8 a 15 Consumo de alto riesgo 16 a 19 Consumo perjudicial. 20 a 40 Probable dependencia.

\section{Copa o trago estándar}

También llamado Unidad de Bebida Estándar (UBE); es utilizado para simplificar la medición del consumo de alcohol. Puede tener un grado leve de inexactitud, pero su nivel de precisión es suficientemente bueno para ser usado en el cálculo del consumo de alcohol (Anderson, Gua \& Colon, 2008).

Cada UBE supone entre 8 y 13 gramos de alcohol puro; con esto se puede medir la cantidad de alcohol puro, no la cantidad de líquido que se bebe. Un hígado sano puede metabolizar alrededor de una UBE cada hora u hora y media, por lo que la OMS recomendó a no superar los 30 gramos en hombres y los 20 gramos en mujeres de alcohol diarios (Unidades de bebida estándar, 2016).

La OMS utiliza los siguientes valores para las bebidas estándar (Babor, HigginsBiddle, Saunders \& Monteiro, 2001):

DEL VALLE-ÁVILA, Paula Carmina \& GARCÍA-RODRÍGUEZ, Juan Carlos. Estudio sobre el consumo de riesgo y perjudicial del alcohol en alumnos de la UT del Norte de Aguascalientes. Revista de Gestión Universitaria. 2019 
- $\quad 330 \mathrm{ml}$ de cerveza al 5\% de alcohol.

- $\quad 140 \mathrm{ml}$ de vino al $12 \%$ de alcohol.

- $\quad 90 \mathrm{ml} \mathrm{de} \mathrm{vinos} \mathrm{fortificados} \mathrm{(por} \mathrm{ejempo}$ jerez) al $18 \%$ de alcohol.

$70 \mathrm{ml}$ de licor o aperitivo al $25 \%$ de alcohol.

$40 \mathrm{ml}$ de bebidas espirituosas al $40 \%$ de alcohol.

A su vez, partiendo de estos valores, Medina (2015), proporciona una tabla de equivalencias en base al número de UBE, copas o tragos estándar:

- Una cerveza de lata: 1 trago o copa estándar.

Una caguama: 3 tragos o copas estándar. Una botella de destilados de 1/4: 6 tragos o copas estándar.

Una botella de destilados de 3/4: 17 tragos o copas estándar.

Una botella de destilados de 1 litro: 22 tragos o copas estándar.

Una botella de vino de 3/4: 5 tragos o copas estándar.

Una botella de vino de 1 litro: 8 tragos o copas estándar.

\section{Consumo riesgoso y dañino de alcohol (CRDA)}

El CRDA se define como un patrón de consumo de bebidas embriagantes, que se sitúa en un continuum de severidad, que coloca al sujeto en riesgo de desarrollar problemas de salud y/o que puede desembocar en francas complicaciones físicas $\quad y / o$ psicológicas (accidentes, victimización, violencia, dependencia al alcohol, etc.) (Díaz, Díaz, Hernández-Ávila, Fernández, Solís \& Narro, 2009).

\section{Metodología}

Se desarrollará un diagnóstico, mediante la aplicación del cuestionario AUDIT, a los estudiantes de la Universidad Tecnológica del Norte de Aguascalientes (UTNA) de segundo cuatrimestre de 14 programas educativos de nivel Técnico Superior Universitario (TSU), con lo cual se obtendrá información concerniente al tipo de consumo de alcohol de dichos estudiantes. a. Procedimiento general de análisis de datos

La información recopilada del diagnóstico, será tratada solamente de forma estadística, utilizando la estadística descriptiva para este fin. Para lograr esto, primeramente se realizará el concentrado de respuestas del cuestionario en una tabla, para de esta forma tener un fácil acceso a la información y facilitar el análisis estadístico.

De la parte de preguntas demográficas, se realizarán gráficas y tablas descriptivas de la información, obteniéndose, principalmente, porcentajes correspondientes a la distribución de la muestra en sexo, lugar de procedencia, edad, ingresos personales y dependencia económica. Esta información se organizará, se tabulará y servirá para realizar gráficos circulares donde se muestren los porcentajes correspondientes a cada sección.

En cuanto a las preguntas del cuestionario AUDIT, estas se evaluarán y se asignará una calificación por cada respuesta, la cual irá del 0 al 4, para al final, obtener la calificación total en cada cuestionario, para enseguida, asignarle un rango distintivo de consumo. Los rangos utilizados por el AUDIT se mencionaron con anterioridad.

Con base a los rangos de consumo, se clasificaron los resultados de cada cuestionario aplicado y se realizó el ordenamiento y agrupación de los resultados, para de esta forma poder construir las gráficas de sectores representativos del tipo de consumo de los estudiantes de la UTNA.

\section{Resultados}

\section{Resultados Globales}

Una vez aplicado el AUDIT, se procedió al análisis de resultados, iniciando por los datos demográficos, los cuales se presentan por medio de gráficas circulares.

De los 756 encuestados, 55\% (416) fueron hombres y el $45 \%$ (340) fueron mujeres. 


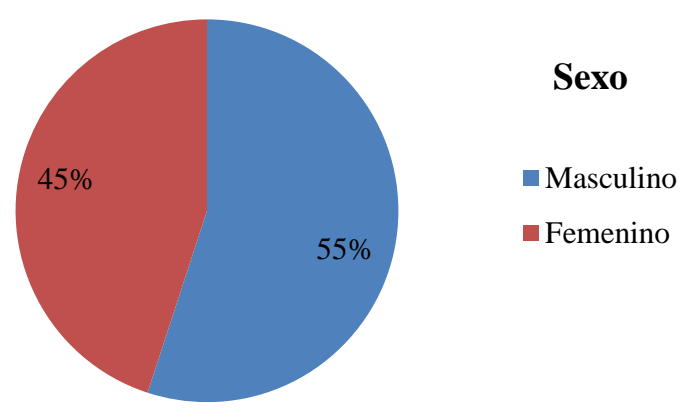

Gráfica 1 Población de estudio por género Fuente: Elaboración Propia

El rango de edad de los encuestados va desde los 17 hasta los 35 años. Siendo que la gran mayoría de los encuestados (87\%) tienen entre los 18 y 19 años.

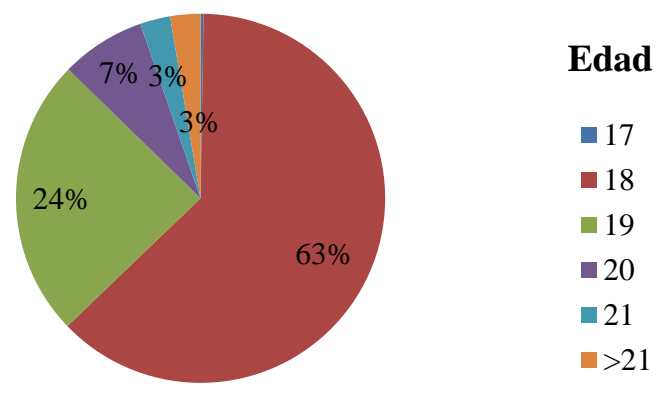

Gráfica 2 Edades de la población de studio Fuente: Elaboración Propia

Casi la mitad de los alumnos de la Universidad de segundo cuatrimestre $(46 \%)$ proviene de los municipios de Rincón de Romos, Pabellón de Arteaga y San Francisco de los Romo; el resto proviene de siete municipios más de Aguascalientes y 7 municipios de Zacatecas.

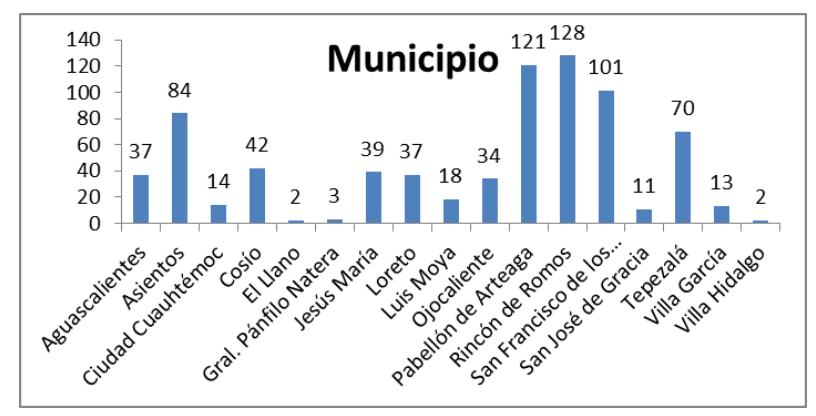

Gráfica 3 Municipio de procedencia de los alumnos de la UTNA

Fuente: Elaboración Propia

En lo referente a ingresos familiares, más del $80 \%$ de los encuestados manifestaron tener un ingreso por debajo de los $\$ 5,000$ mensuales, mientras que solamente 10 encuestados cuentan con ingresos familiares superiores a los $\$ 15,000$ mensuales.

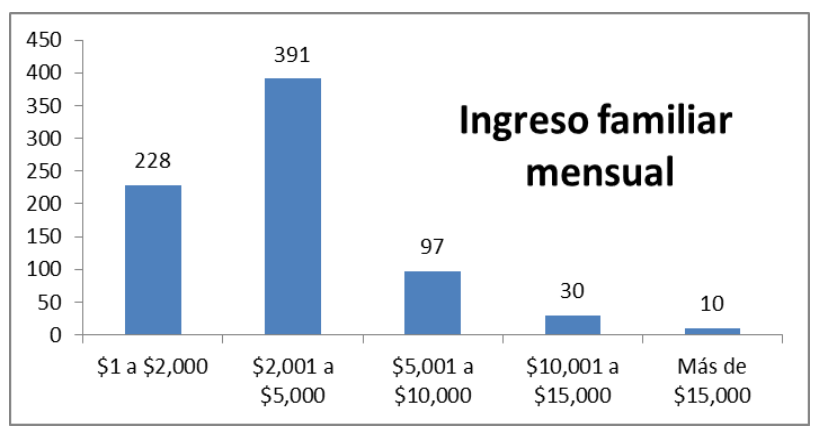

Gráfica 4 Ingreso familiar mensual Fuente: Elaboración Propia

Una gran mayoría de los estudiantes, casi el $90 \%$, dependen económicamente directamente de sus padres, para cubrir los gastos de sus estudios.

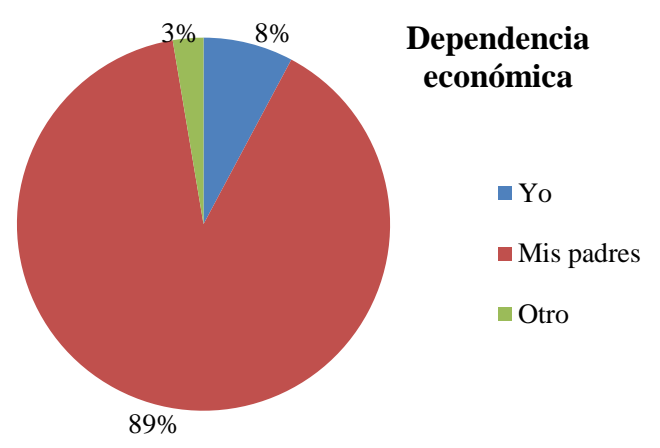

Gráfica 5 Dependencia económica Fuente: Elaboración Propia

Finalmente, la parte fundamental del diagnóstico, lo relacionado con el consumo de alcohol; se encontraron 120 encuestados con un consumo de alto riesgo, 29 con un consumo perjudicial y 14 encuestados con una probable dependencia al alcohol, es decir, $21.56 \%$ de los encuestados presenta un consumo de alcohol que requiere la atención del mismo, ya que esto les puede generar problemas de tipo académico, social y personal.

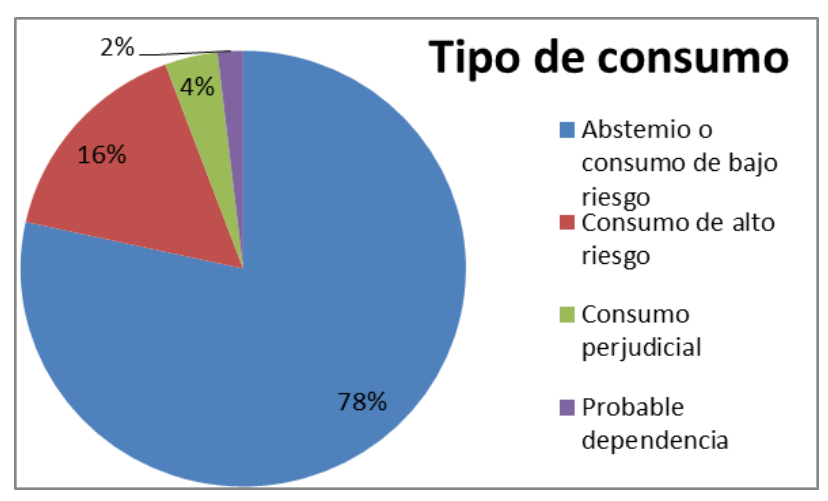

Gráfica 6 Tipo de consumo general

Fuente: Elaboración Propia 


\section{Resultados del tipo de consumo por carrea}

a) TSU en Administración área Capital Humano.

Se aplicaron en total 84 cuestionarios en esta carrera, de los cuales, 20 encuestados tienen un consumo de alcohol de alto riesgo o perjudicial, y de esos veinte, dos presentan evidencia de una probable dependencia al alcohol.

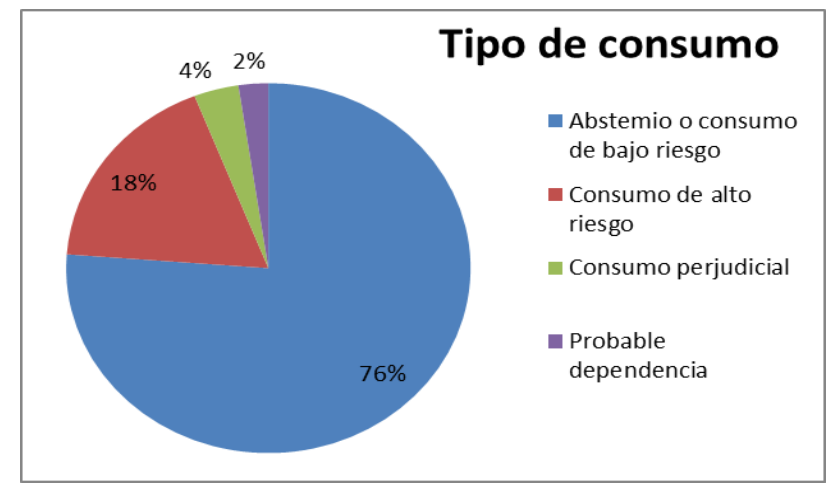

Gráfica 7 Tipo de consumo ACH

Fuente: Elaboración Propia

b) TSU en Administración área Formulación y Evaluación de Proyectos

De este programa educativo, contestaron el cuestionario un total de 48 estudiantes, de los cuales, el $13 \%$ de los estudiantes (7) tiene un consumo de alto riesgo o perjudicial.

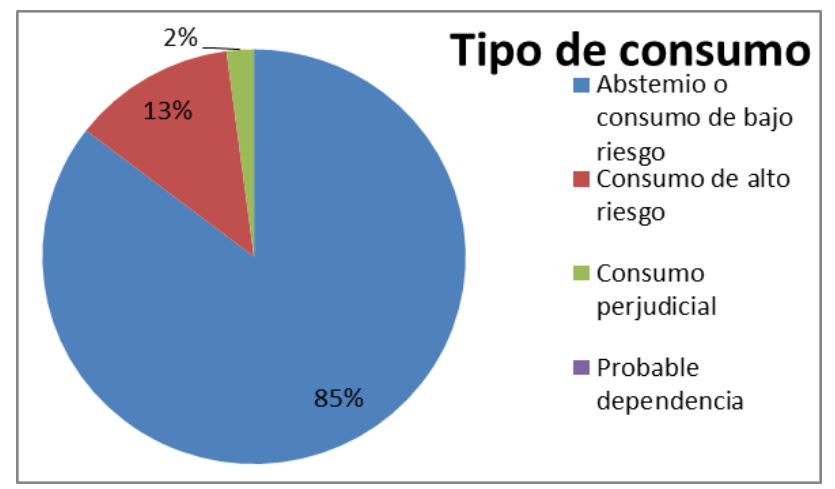

Gráfica 8 Tipo de consumo AFEP

Fuente: Elaboración Propia

\section{c) TSU en Contaduría}

Se encuestaron un total de 71 estudiantes, de los cuales, el $16 \%$ de los encuestados presenta evidencia de un consumo de alto riesgo o consumo perjudicial; de ese porcentaje, hay dos estudiantes que presentan evidencia de una probable dependencia al alcohol.

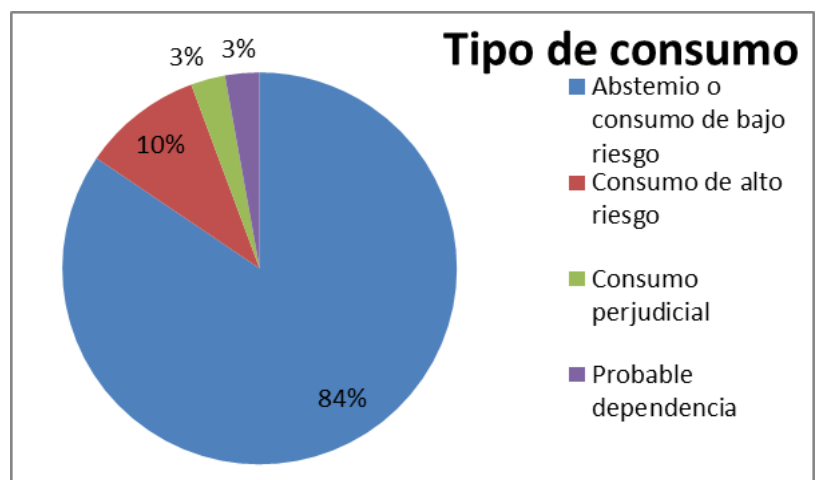

Gráfica 9 Tipo de consumo CON

Fuente: Elaboración Propia

d) TSU en Agricultura Sustentable y Protegida

Del programa educativo de Agricultura Sustentable y Protegida, en total se tienen 33 encuestas, de las cuales, siete alumnos presentan un consumo de alto riesgo y un alumno más presenta evidencia una probable dependencia al alcohol.

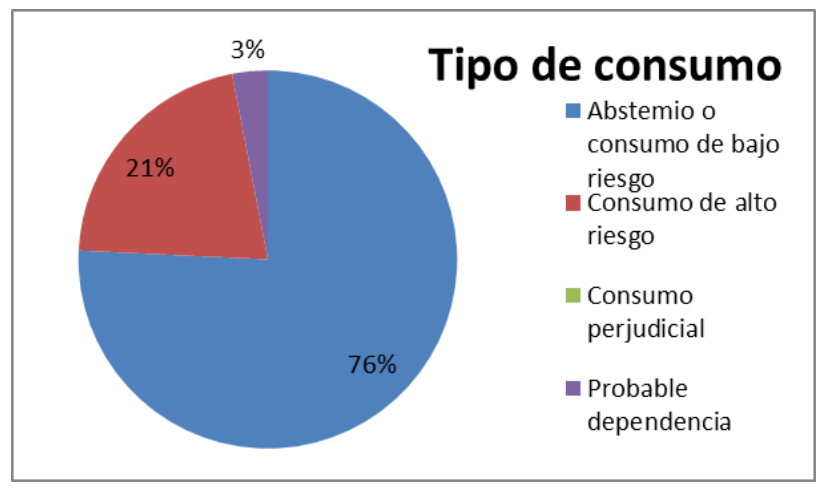

Gráfica 10 Tipo de consumo ASyP

Fuente: Elaboración Propia

e) TSU en Desarrollo de Negoción área Mercadotecnia

Se aplicaron un total de 43 cuestionarios, de los cuales, seis alumnos presentan consumo de alto riesgo o perjudicial, mientras que un alumno presenta evidencia de una probable dependencia al alcohol. 


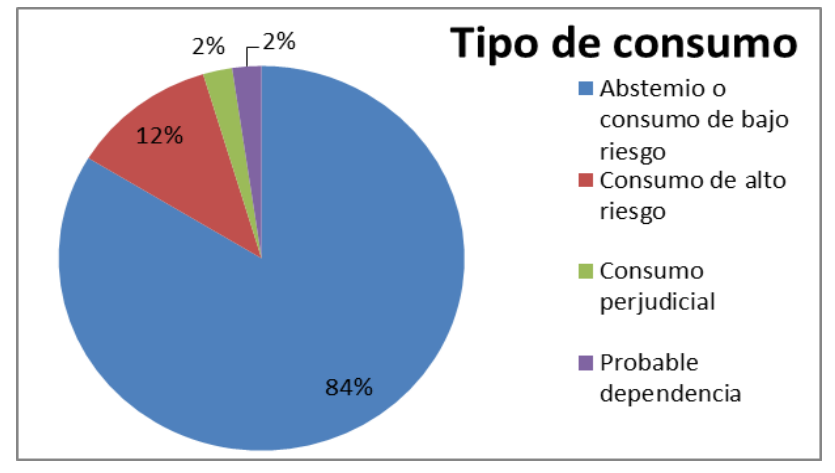

Gráfica 11 Tipo de consumo DNM Fuente: Elaboración Propia

f) TSU en Logística área Cadena de Suministros

En el programa educativo de Logística área Cadena de Suministros se aplicaron 52 encuestas, de las cuales, se tienen 12 alumnos con un consumo de alcohol de alto riesgo; además, se tienen dos alumnos con evidencia de una probable dependencia al alcohol.

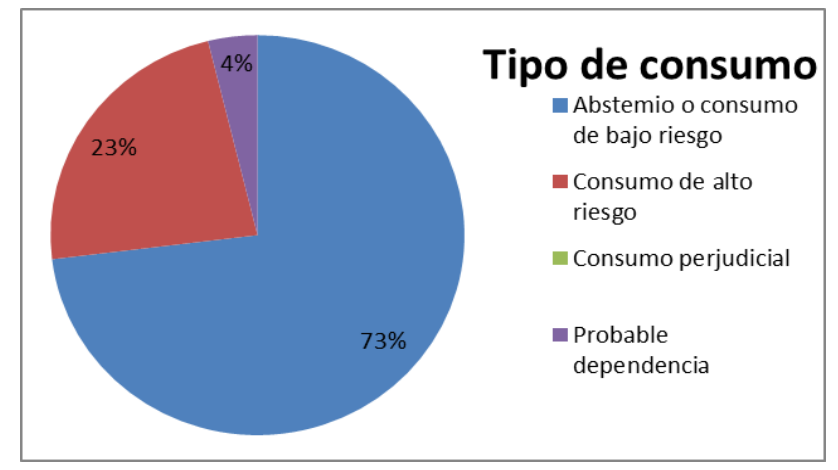

Gráfica 12 Tipo de consumo LCS

Fuente: Elaboración Propia

\section{g) TSU en Mecánica área Automotriz}

En el programa educativo de TSU en Mecánica área Automotriz se aplicaron 54 cuestionarios, de los cuales, el 30\% de los encuestados presenta un consumo de alcohol de alto riesgo o perjudicial; dentro de ese mismo porcentaje, se encuentra un alumno con una probable dependencia al alcohol.
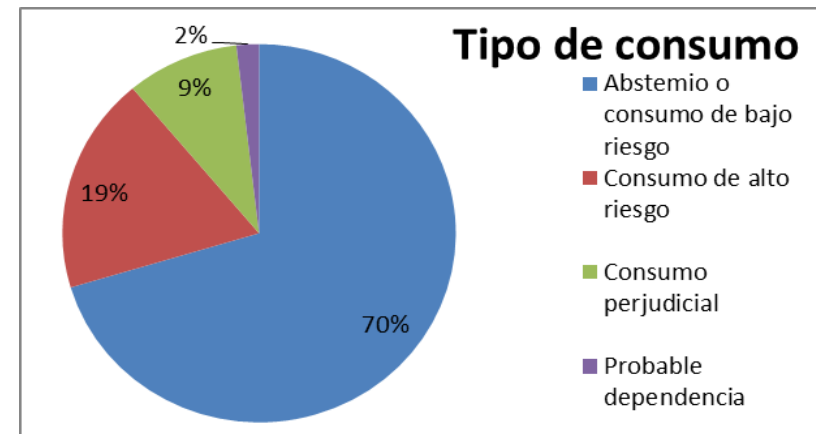

Gráfica 13 Tipo de consumo MA

Fuente: Elaboración Propia

ISSN 2523-2495

ECORFAN® Todos los derechos reservados

\section{h) TSU en Mantenimiento Industrial}

Se recopilaron un total de 88 cuestionarios de este programa educativo, de los cuales, 26 alumnos presentan un consumo de alto riesgo o perjudicial; de ellos, 3 estudiantes presentan evidencia de una probable dependencia al alcohol.

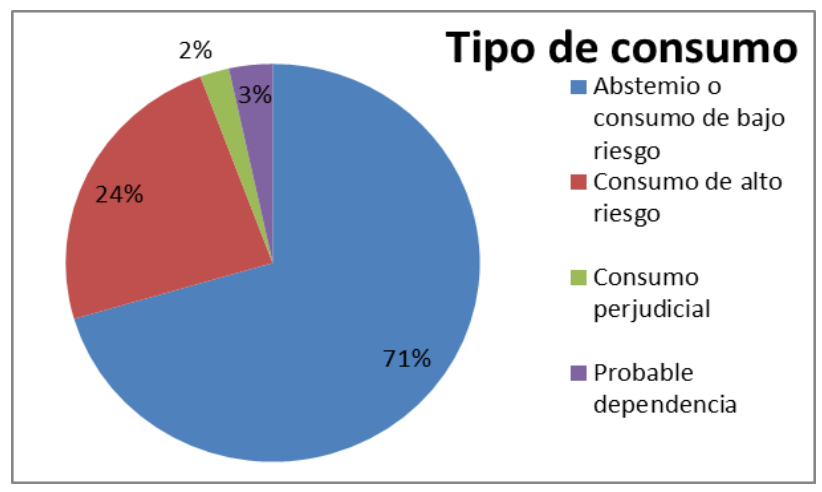

Gráfica 14 Tipo de consumo MI

Fuente: Elaboración Propia

i) TSU en Procesos Industriales área Automotriz.

En total, 36 personas del programa educativo de Procesos Industriales área Automotriz contestaron el cuestionario, de los cuales, un total de ocho estudiantes del programa educativo presentan evidencia de un consumo de alto riesgo o de un consumo perjudicial.

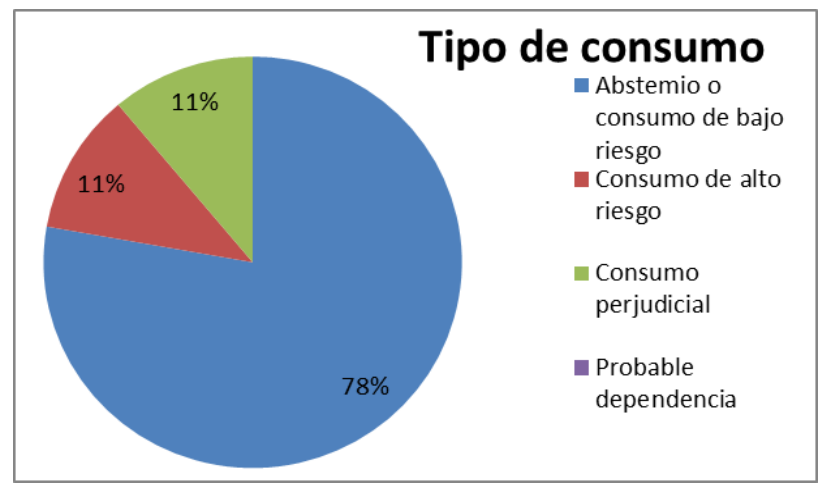

Gráfica 15 Tipo de consumo PIA

Fuente: Elaboración Propia

i)

TSU en Procesos Industriales área
Manufactura

Del programa educativo de Procesos Industriales área Manufactura se recogieron 57 cuestionarios, de los cuales, 11 estudiantes del programa educativo presentan evidencia de un consumo de alto riesgo o perjudicial, además, hay un alumno que presenta una probable dependencia al alcohol. 


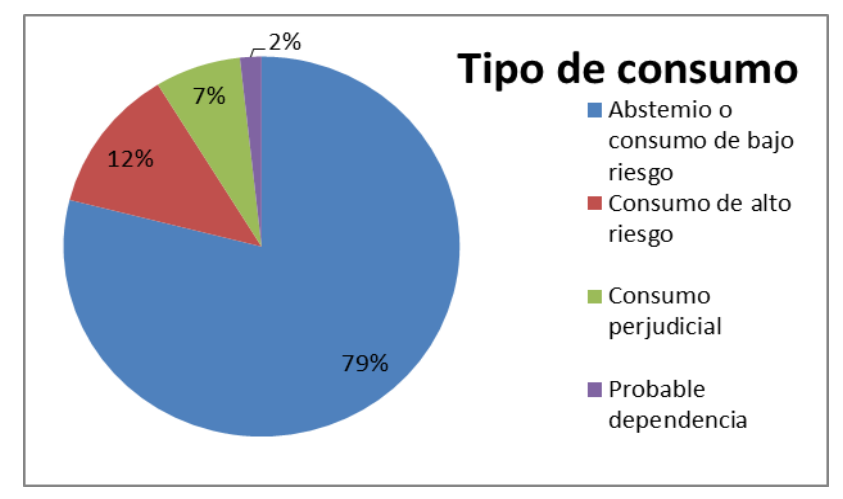

Gráfica 16 Tipo de consumo PIM Fuente: Elaboración Propia

j) TSU en Mecatrónica área

Se recabaron 69 cuestionarios en el programa educativo de Mecatrónica área automatización, de los cuales, 17 alumnos del programa educativo presentan evidencia de un consumo de alto riesgo o consumo perjudicial.

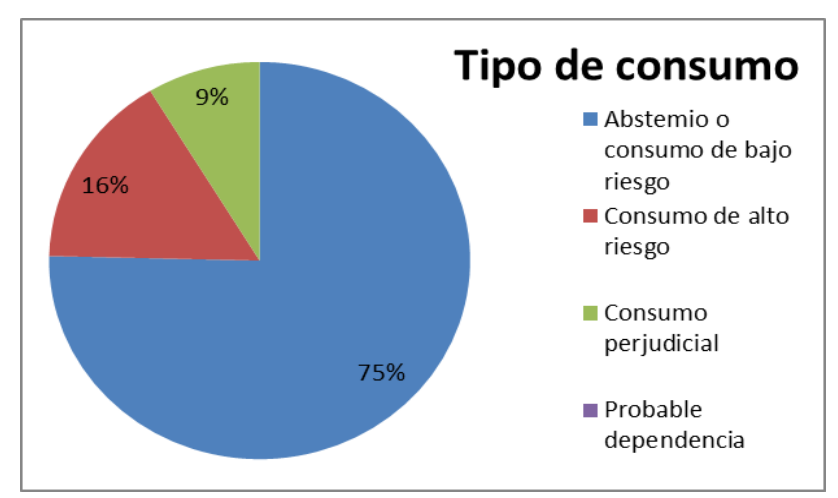

Gráfica 17 Tipo de consumo MTAUT

Fuente: Elaboración Propia

k) TSU en Mecatrónica área Sistemas de Manufactura Flexible

Se reunieron 43 cuestionarios del programa de educativo de Mecatrónica área Sistemas de Manufactura Flexible, de los cuales, se encontraron a siete estudiantes con un consumo de alto riesgo o un consumo perjudicial de alcohol.

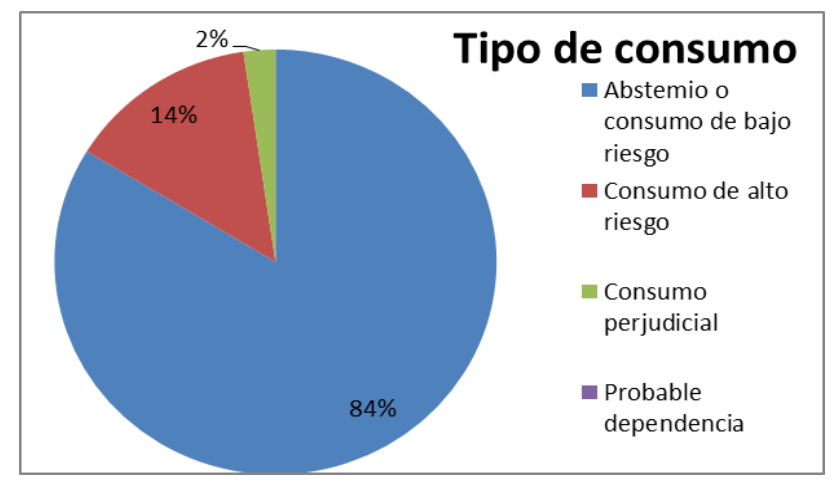

Gráfico 18 Tipo de consumo MTSMF

Fuente: Elaboración Propia

ISSN 2523-2495

ECORFAN® Todos los derechos reservados
1) TSU en Tecnologías de la Información área Desarrollo de Software Multiplataforma

Se obtuvieron 59 encuestas de este programa educativo, de las cuales, se encontró que seis alumnos presentan un consumo de alcohol de alto riesgo. Mientras que el 2\% presentó un consumo perjudicial.

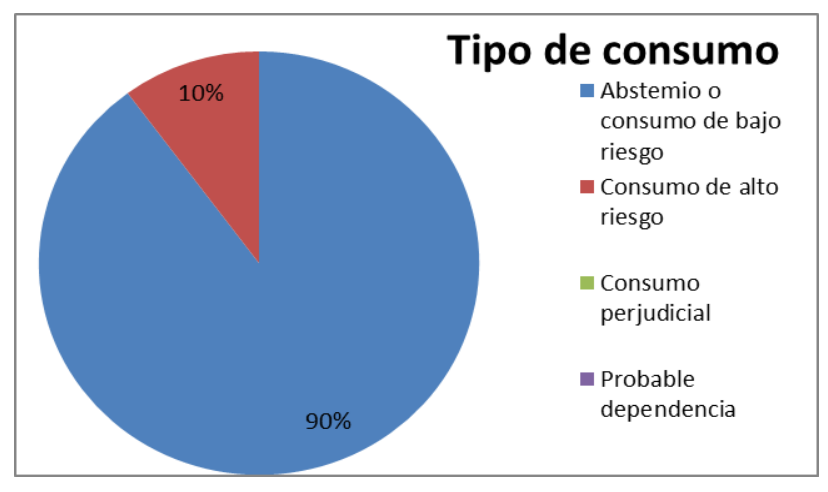

Gráfica 19 Tipo de consumo TIDSM

Fuente: Elaboración Propia

m) TSU en Tecnologías de la Información área Entornos Virtuales y Negocios Digitales

Se aplicaron un total de 19 cuestionarios, de los cuales, se encontró con que tres alumnos presentan un consumo de alto riesgo, mientras que un estudiante del programa educativo tiene una probable dependencia al alcohol.

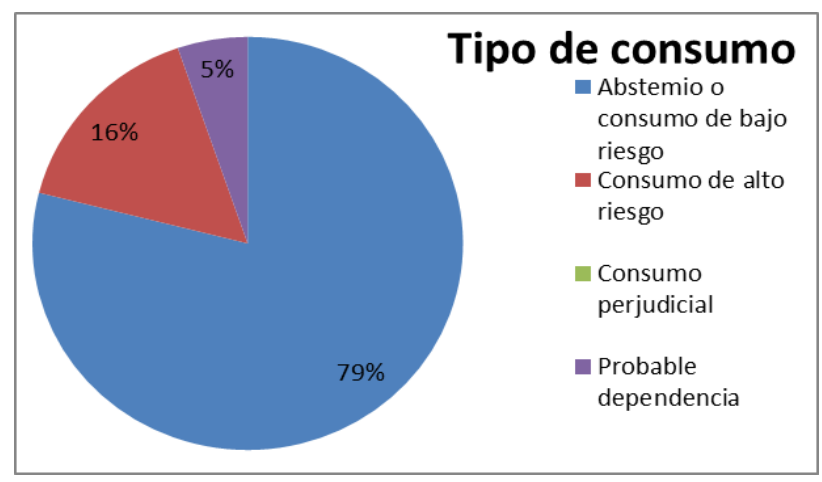

Gráfico 20 Tipo de consumo TIEVND

Fuente: Elaboración Propia

\section{Conclusiones}

A la vista de los resultados obtenidos en el cuestionario, es necesario realizar una concientización en los jóvenes estudiantes de la carrera del nivel de TSU de la UTNA, pues los resultados del cuestionario AUDIT señalan que varios de ellos se encuentran en un consumo potencialmente peligroso para ellos. 
Este tipo de consumo les puede afectar y acarrear consecuencias negativas en cuestiones relacionadas a su vida académica (bajo desempeño escolar, rezago y/o deserción), social (violencia, inestabilidad laboral, falta de oportunidades, etc.) y personal (daños y secuelas físicas y mentales).

El poder realizar algún tipo de actividad que concientice a los jóvenes en lo relacionado a su consumo de alcohol, disminuirá las probabilidades de que este les afecte en cualquier aspecto de su vida; esto convierte al combate a las adicciones (el alcohol entre ellas) en uno de los temas transversales que deben de ser atendidos de forma inmediata dentro del contexto de la educación superior, ya que el consumo de alcohol genera otro tipo de situaciones problemáticas y puede llevar al consumidor a consecuencias fatales.

El poder trabajar con un tema que ha sido ampliamente investigado, permite el poder evaluar y valorar distintos tipos de instrumentos (ya validados), para poder realizar diagnósticos e intervenciones sobre el tema que interesa.

El problema del alcoholismo en jóvenes ha tenido un aumento en los últimos años, lo cual provoca que estos se vean afectados en los ámbitos personal, social, educativo y económico. Es necesario concientizar a los jóvenes sobre estos problemas y a su vez, motivarlos a hacerlos participes de programas que los ayuden a superar los problemas de adicciones.

\section{Agradecimiento}

Al área de Asesoría Psicopedagógica y la Coordinación de Tutorías de la Universidad Tecnológica del Norte de Aguascalientes por el apoyo brindado en la recolección de datos.

\section{Referencias}

Alcohólicos Anónimos, A.C. (2012). Alcoholismo. Recuperado el 4 de julio de 2016, de Central Mexicana de Servicios Generales de Alcohólicos Anónimos, A.C.: http://www.aamexico.org.mx/Alcoholismo.html

Alvarado, S. (5 de julio de 2016). Definición de adicción. Obtenido de Adcciones: http://www.adicciones.org/enfermedad/definici on.html
Bolet, M. (julio-agosto de 2000). La prevención del alcoholismo en los adolescentes. Recuperado el 4 de julio de 2016, de Revista Cubina de Medicina General Integral versión On-Line: http://scielo.sld.cu/scielo.php?pid=S086421252000000400019\&script=sci_arttext\&tlng= en

Gobierno del estado de Aguascalientes. (2009). Gobierno del Estado de Aguascalientes. Recuperado el 1 de abril de 2009, de Actividad económica: http://www.aguascalientes.gob.mx

Gobierno del Estado de Aguascalientes. (2009). Gobierno del Estado de Aguascalientes. Recuperado el 30 de marzo de 2009, de Rincón de Romos: http://www.aguascalientes.gob.mx

Hernández Sampieri, R., Fernández Collado, C., \& Baptista Lucio, M. (2010). Metodología de la investigación (5a ed.). México, D.F.: Mc GrawHill.

Instituto Mexicano del Seguro Social. (15 de julio de 2015). Alcoholismo. Recuperado el 4 de julio de 2016, de Salud en línea: http://www.imss.gob.mx/salud-en-

linea/alcoholismo

Instituto Nacional de Psiquiatría Ramón de la Fuente Muñiz; Instituto Nacional de Salud Pública; Secretaría de Salud. (2012). Encuesta Nacional de Adicciones 2011: Reporte de Alcohol. Recuperado el 3 de julio de 2016, de http://www.conadic.salud.gob.mx/pdfs/ENA_2 011_ALCOHOL.pdf

La Jornada de Aguascalientes. (2017). Consumo de Alcohol en Aguascalientes. Recuperado el 20 de 08 de 2019, de La Jornada de Aguascalientes: https://www.lja.mx/2017/02/en-aguascalientesconsumo-alcohol-comienza-en-del-80-losjovenes-14-20-anos/

Lucio, E., Gómez, H., Morales, B., \& Pérez, M. (2009). Uso del AUDIT y el DAST-10 para la identificación de abuso de sustancias psicoactivas y alcohol en adolescentes. Revista Colombiana de Psicología, 9-17.

Medina Camacho, O. (abril de 2015). Habilidades Básicas de promoción de salud en Tutoría. 116. Aguascalientes, México. 
OMS. (Enero de 2015). Alcohol. Recuperado el 3 de julio de 2016, de Organizacion Mundial de la

Salud:

http://www.who.int/mediacentre/factsheets/fs34 9/es/

Otaño, Y., \& Valdes, Y. (septiembre-diciembre de 2004). Algunas reflexiones sobre el alcoholismo en la comunidad. Recuperado el 4 de julio de 2016, de Revista Cubana de Enfermería On-line: http://scielo.sld.cu/scielo.php?pid=S086403192004000300003\&script=sci_arttext\&tlng= en

Rodríguez, P. (2019). A los 10 años inicia el consumo de alcohol en México: Conadic. Recuperado el 19 de 08 de 2019, de Excelsior: https://www.excelsior.com.mx/nacional/a-los10-anos-se-inicia-el-consumo-de-alcohol-enmexico-conadic/1290174

Secretaría de la Juventud del Estado de Aguascalientes. (2014). Consulta Estatal de la Juventud 2014. Recuperado el 3 de julio de 2016, de Servicio Estatal de Información Estadística y Geográfica: www.aguascalientes.gob.mx/CEPLAP/SEIEG/c uadros/SEIEG-1740.docx

Secretaría de Salud. (2017). Encuesta Nacional de Consumo de Alcohol. Droga y Tabaco 20162017: Reporte de Alcohol. Cd. de México: Secretaría de Salud. 